\title{
CONTROL EFFECTS OF PERFORATED LINERS ON THE COMBUSTION INSTABILITY IN AN ANNULAR COMBUSTOR
}

\author{
Guangyu Zhang \\ Beihang University \\ Guangyu.zhang@buaa.edu.cn \\ Beijing, China \\ Xiaodong Jing \\ Beihang University \\ jingxd@buaa.edu.cn \\ Beijing, China
}

\author{
Xiaoyu Wang \\ Beihang University \\ bhwxy@buaa.edu.cn \\ Beijing, China \\ Xiaofeng Sun \\ Beihang University \\ sunxf@buaa.edu.cn \\ Beijing, China
}

\begin{abstract}
Perforated liners with bias flow are efficient and robust in damping acoustic waves as well as cooling combustor walls in combustion systems. And many analytical models with the superiority of being physically clear and time-costless have been developed to describe the acoustic response of perforated liner with bias flow. Considering that the issue of perforated liners lined in both the inner and outer walls of the annular combustor is important. In this paper, we have applied transfer element method to describe the acoustics in the lined section that is influenced by both of the outer and inner perforated liners, and established the eigenvalue problem combined with appropriate matching and boundary conditions. It was found that the locations of the perforated liners are crucial to the control effect. In particular, when the homogeneous perforated liners nearly take up the whole length of the combustion chamber, the control effect is insignificant. This is mainly due to the fact that when the length of the perforated liner with backing cavity is equal to the combustion chamber, the mode shape in the backing cavity is similar to that in the combustion chamber at the longitudinal resonance frequency, leading to unconspicuous pressure fluctuation difference across the perforated plates that is responsible for the damping process. Moreover, we found that by splitting the perforated liner axially into two individual liners, the control effect was improved.
\end{abstract}

\section{INTRODUCTION}

With more stringent environmental requirements for aero-engines, the adoption of lean premixed and prevaporized (LPP) combustors that have low $N O_{x}$ emissions becomes a mainstream in modern aero-engines. However, this kind of engine is more susceptible to combustion instability that is a result of coupling between unsteady heat release and acoustic waves. This may make the combustor suffer severe damage immediately and end up with disastrous failure. Therefore, significant work has been devoted to the study on the mechanism and control methods of combustion instabilities (Lieuwen, 2005; Poinsot, 2017). Worth and Dawson (Worth, 2013) studied the global flame dynamics and self-excited azimuthal mode of a model annular combustor with emphasis on the effect of flame separation distances on the instability characteristics and flame dynamics. Bourgouin and coworkers (Bourgouin, 2013) designed a laboratory scale annular combustor, and reported the flame dynamics in the observed longitudinal and azimuthal unstable modes.

In order to minimize damages resulting from the combustion instability, perforated liners are very popular in practical applications (Eldredge and Dowling, 2003; Zhao, 2015). In particular, perforated liners with bias flow take effect by allowing cooling air through the combustor walls to prevent the combustor walls from burning out, and additionally, damping acoustic waves by conversion of acoustic energy into vortical motions at the edges of the plates' holes (Howe, 1979; Jing and Sun, 1999) in modern combustion systems. Eldredge and Dowling (Eldredge and Dowling, 2003) carried out both of theoretical and experimental investigations on the effectiveness of a cylindrical perforated liner with mean bias flow in its absorption of planar waves in a duct. For the consideration of the interactions between the perforated liner and the other components in the combustor, it is a desirable and meaningful attempt to put the perforated liner in a thermoacoustic system, analysing the instability of the system including the interactions between the perforated liners and acoustic waves emitted by the unsteady heat release with analytical methods. 
The present research concerns mainly on the effects of perforated plates with backing cavity on controlling combustion instability theoretically. We have applied transfer element method (TEM) (Sun et al., 2008, Wang and Sun, 2011) to describe the interactions between the perforated liners lined in both inner and outer walls of an annular combustion chamber and acoustics in the pipe. In the next section, the model that consists of pressure fluctuation description and matching conditions, will be briefly introduced. Then, some calculation results about the control effects of perforated liners are presented, following which the improvement technique of the single perforated liner that occupies the whole length of the chamber is given. Lastly, conclusion is made.

\section{MODEL}

The geometry to be investigated in the present work is a simplified premixed annular combustor that consists of 20 premixers and one combustion chamber. The plenum before the premixers, which is often decoupled with the combustion chamber in eigen mode calculations, is ignored for simplicity. The simplified annular combustor is totally asymmetric, and the half section view of the geometry is shown in Figure 1. On both of the inner and outer walls of the combustion chamber, two perforated plates with backing cavities are respectively installed.

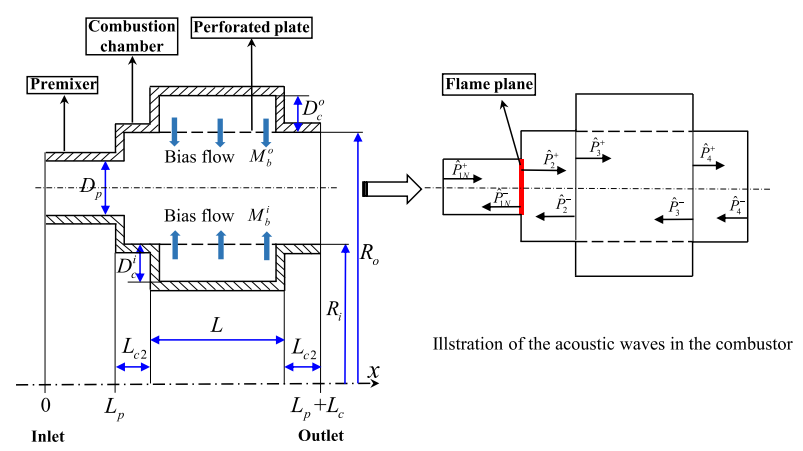

Figure 1 (Color online) Illustration of the half section view of the geometry of the simplified annular combustor.

The length and diameter of the premixer are respectively denoted by $L_{p}$ and $D_{p}$. The inner and outer radii of the combustion chamber are respectively denoted by $R_{i}$ and $R_{o}$. The Mach number of the bias flow is denoted by $M_{b}$. And the depth of the inner and outer backing cavity are represented by $D_{c}^{i}$ and $D_{c}^{o}$. The flames are assumed to be modelled by flame plane and located just the exit of the premixers, as shown in Figure 1.

The premixers are so thin that the acoustic waves inside can be described by plane waves:

$$
\begin{gathered}
p_{1 N}^{\prime}(x, t)=\left(P_{1 N}^{+} e^{\frac{-i \omega t}{\bar{c}_{1}+U_{1}}}+P_{1 N}^{+} e^{\frac{-i \omega t}{\bar{c}_{1}+U_{1}}}\right) e^{i \omega t} \\
u_{1 N}^{\prime}(x, t)=\frac{1}{\bar{\rho}_{1} \bar{c}_{1}}\left(P_{1 N}^{+} e^{\frac{-i \omega t}{\bar{c}_{1}+U_{1}}}-P_{1 N}^{+} e^{\frac{-i \omega t}{\bar{c}_{1}+U_{1}}}\right) e^{i \omega t}
\end{gathered}
$$

where $p$ represents the pressure, and $u$ the velocity. The prime denotes the fluctuation component, and overline the mean component. $P_{1 N}^{+}$and $P_{1 N}^{-}$respectively denote the complex amplitudes of downstream and upstream propagating waves, $\omega$ the complex angular frequency. $N$ denotes the sequence number of the premixers from 1 to $20 . \bar{\rho}_{1}$ and $\bar{c}_{1}$ are the mean density and sound speed in the premixers, respectively. $U_{1}$ is the mean flow velocity in the premixers.

In the present work, we neglect the convective terms such as the vorticity and entropy waves. The acoustic waves in the combustor with rigid wall boundary condition are threedimensional which satisfy the wave equation:

$$
\frac{1}{\bar{c}_{2}^{2}} \frac{D^{2}}{D t^{2}} p^{\prime}-\nabla^{2} p^{\prime}=0,
$$

where $\bar{c}_{2}$ denotes the mean sound speed in the combustor.

By using separation of variables method, the pressure fluctuation component is obtained

$$
\begin{aligned}
& p_{(2,4)}^{\prime}(x, \theta, r, t)=\sum_{m=-\infty}^{+\infty} \sum_{n=1}^{+\infty} \psi\left(z_{m n} r\right) \\
& \times\left(P_{(2,4) m n}^{+} e^{i k_{m n}^{+} x}+P_{(2,4) m n}^{-} e^{i k_{m n}^{-}\left(x-L_{c(2.4)}\right)}\right) e^{i(m \theta+\omega t)},
\end{aligned}
$$

where $\psi$ is the eigenfunction of the annular combustion chamber. $m$ and $n$ are circumferential and radial mode numbers, correspondingly. $k_{m n}^{ \pm}$are the axial wavenumbers of downstream and upstream propagating acoustic waves. The axial acoustic velocities of the rigid wall sections can be obtained according to the momentum equation, the detailed description of the waves in the rigid-wall sections can be referred in Ref. (Zhang et al., 2017).

The acoustic waves in the lined section can be described by transfer element method (TEM) (Sun et al., 2008, Wang and Sun, 2011), the acoustic waves are described as a sum of incident waves with rigid wall boundary condition and disturbance waves radiated by the perforated plates. More of this idea can be referred in Ref (Namba and Fukushige, 1980; Sun et al., 2008). In the present work, the response of the perforated plates with bias flow is described by the Rayleigh conductivity. More details can be found in Ref. (Eldredge and Dowling, 2003).

The inlet boundary condition for the premixers can be set up as (Akamatsu and Dowling, 2011):

$$
u_{1 N}^{\prime}=-\varsigma p_{1 N}^{\prime}, x=0 \text {, }
$$

where $\varsigma$ is a constant determined by the discharge coefficient of the premixers.

The outlet of the combustor is choked, and can be approximated to be a closed end when the Mach number is low, then we obtain the outlet boundary condition for the annular combustion chamber:

$$
u_{4}^{\prime}=0, x=L_{p}+L_{c} .
$$

Since the Mach number is low, the momentum conservation across the interface of the premixers and the combustion chamber can be approximated to

$$
p_{1 N}^{\prime}=p_{2}^{\prime},(x, r, \theta)=\left(L_{p}, R, \theta_{N}\right)
$$

We suppose that the combustion zone is nearly isobaric, the combustion flame is compact and takes place at the exit of 
the premixers. Then the energy conservation across the combustion flame can be reduced to

$$
\frac{\gamma}{\gamma-1} \frac{\partial}{\partial x_{j}}\left(\bar{p} u_{j}^{\prime}\right)=q^{\prime}
$$

By virtue of orthogonality of the eigenfunctions of the annular combustion chamber and asymmetry of the geometry, Eq. (8) is multiplied by the weighting function to separate the azimuthal mode $M$ to be investigated

$$
W=e^{-i M \theta} \psi_{M n^{\prime}}\left(z_{M n^{\prime}} r\right) \text {. }
$$

To obtain one individual circumferential mode $m$ for investigation. By integrating Eq. (8) over a tiny distance $\mathrm{d} x$, we have

$$
\frac{\gamma W}{\gamma-1}\left[\bar{p}_{2} u_{2}^{\prime}\right]_{x=L_{p}^{+}}-\frac{\gamma W}{\gamma-1}\left[\bar{p}_{2} u_{2}^{\prime}\right]_{x=L_{p}^{-}}=\left[q^{\prime} W\right],
$$

where $L_{p}^{+}$and $L_{p}^{+}$denote axial locations just after and before the premix duct outlet, respectively. The square brackets denote the area integration. We simply adopt the flame transfer function that has been used in Ref. (Eldredge and Dowling, 2003). The unsteady heat release generated by the flame after each premixer can be represented by

$$
\hat{q}_{N}=-k_{f} \bar{q}_{N} \frac{\hat{u}_{1 N}}{U_{1}},
$$

where $k_{f}$ is the gain of the flame transfer function, it is assumed to be unity in the present study. $\hat{q}_{N}$ denotes the complex amplitude of the unsteady heat release of the $\mathrm{N}$-th premixer.

For the matching conditions at the leading edge and trailing edge of the perforated liners, since the Mach number in the chamber is very low, it is reasonable to assume the continuity of acoustic pressure and velocity

$$
\begin{aligned}
& p_{(2,3)}^{\prime}=p_{(3,4)}^{\prime}, \text { at } x=L_{p}+L_{c 1} \text { or } x=L_{p}+L_{c 1}+L, \\
& u_{(2,3)}^{\prime}=u_{(3,4)}^{\prime}, \text { at } x=L_{p}+L_{c 1} \text { or } x=L_{p}+L_{c 1}+L,
\end{aligned}
$$

Till now, the matching conditions for the geometrical model in Figure 1 have been presented through Eqs. (5), (6), (7), (10), (12) and (13). With the unknown amplitudes of $P_{1 N}^{+}, P_{1 N}^{-}, P_{2 m n}^{+}, P_{2 m n}^{-}, P_{3 m n}^{+}, P_{3 m n}^{-}, P_{4 m n}^{+}$and $P_{4 m n}^{-}$. It can be represented by the equation

$$
\mathbf{X P}=\mathbf{0},
$$

where $\mathbf{X}$ is the coefficient matrix of the equations, and $\mathbf{P}$ denote the unknown amplitudes. Then the eigenfrequency and modeshape can be obtained by solving

$$
\operatorname{det}|\mathbf{X}|=0 \text {. }
$$

\section{RESULTS AND DISCUSSION}

In this section, the effects of the perforated liners on controlling of the combustion instability are theoretically studied by the present model. Emphasis remains on the impact brought by the variation of the length of the perforated liners. Acoustic energy is also considered to both validate of the eigenvalue calculation and figure out the characteristics of acoustic absorption processes. The pressure and mass flux in the premixers are respectively set to be $4 \mathrm{MPa}$ and $50 \mathrm{~kg} / \mathrm{s}$. The temperature in the premixers is taken to be $600 \mathrm{~K}$, and raised to be $2000 \mathrm{~K}$ in the combustor. The length and diameter of the premixer are $0.06 \mathrm{~m}$ and $0.045 \mathrm{~m}$, respectively. The mean area and length of the combustion chamber are $0.3 \mathrm{~m}^{2}$ and $0.3 \mathrm{~m}$, respectively. The depth of the inner and outer backing cavity is set to be $0.03 \mathrm{~m}$. The default geometrical parameters of the perforated plates are shown in Table 1 .

Table 1. Geometrical parameters for the perforated plates.

\begin{tabular}{lcc}
\hline Parameters & Symbols & Values \\
\hline Perforation ratio & $\sigma$ & 0.02 \\
Thickness of perforated plate & $t_{w}$ & $0.8 \times 10^{-3} \mathrm{~m}$ \\
Diameter of the aperture & $d$ & $2 \times 10^{-3} \mathrm{~m}$ \\
Mach number of the bias flow & $M_{b}$ & 0.005 \\
\hline
\end{tabular}

The control effects were checked by examining the eigenfrequencies obtained by solving Eq. (15). The real part of the complex eigenfrequency represents the natural frequency of the system, while the negative imaginary part the growth rate. We only present the results of the first longitudinal and azimuthal mixed mode (Mode 1L1A, where "L" stands for longitudinal and "A" stands for azimuthal). The natural frequency and growth rate as well as the total acoustic energy flux gained versus the length of the perforated liners are shown in Figure 2. The total energy gained is denoted by $E_{t}$, which accounts for all the components in the combustor that can be calculated by the equation given in Ref. (Morfey, 1971),

$$
\left\langle N_{n}\right\rangle=\left\langle p^{\prime} v_{n}^{\prime}\right\rangle=\frac{1}{2} \operatorname{Re}\left(\hat{p} \hat{v}_{n}^{*}\right)
$$

where "*" denotes the conjugate, and the pointy brackets denote the time-mean value.

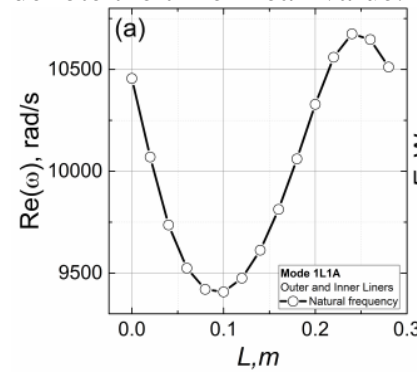

(a)

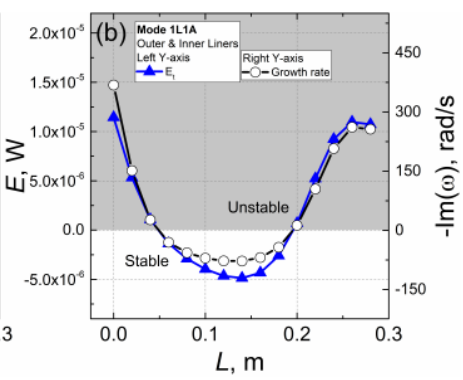

(b)
Figure 2. (Color online) Mode 1L1A; Lc1=0.01 m, L=0.14 m; (a) Natural frequency against the length of perforated liners; (b) growth rate and total acoustic energy flux against the length of perforated liners.

It is observed that when the length of the perforated plates reaches a value that is about $0.14 \mathrm{~m}$, the control effect is the best, and then becomes worse as the length keeps increasing. Take " $L=0.14 \mathrm{~m}$ " and " $L=0.28 \mathrm{~m}$ " for example, the mode shape and acoustic velocity oscillations as well as the acoustic energy flux absorption by the perforated plates per unit area are shown in Figure 3 and Figure 4, respectively. " $e_{i} "$ and " $e_{o}$ " respectively denote the acoustic energy flux absorption by the inner and outer perforated plates per unit area, and can be calculated using Eq. (16). $\hat{p}_{3}^{-}$represents the complex amplitude of the pressure fluctuation in the combustion chamber in the lined section, while $\hat{p}_{d}^{+o}$ and $\hat{p}_{d}^{+i}$ respectively represent the values in the outer and inner 
backing cavities. Comparison of Figure 3 and Figure 4 shows that when $\mathrm{L}=0.28 \mathrm{~m}$, which means that the perforated plates nearly takes up the whole combustion chamber, the pressure mode shape in the backing cavities is nearly the same with that in the combustion chamber, leading to very small acoustic velocity oscillations that results in a small quantity of acoustic energy flux absorption. In the next subsection, it is presented that when the long perforated plates with backing cavities, with length of $0.28 \mathrm{~m}$, are axially split into two individual perforated plates with backing cavities, the control effect will be improved.

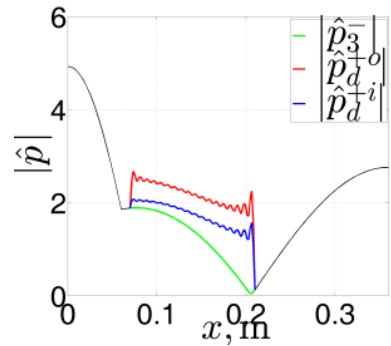

(a)

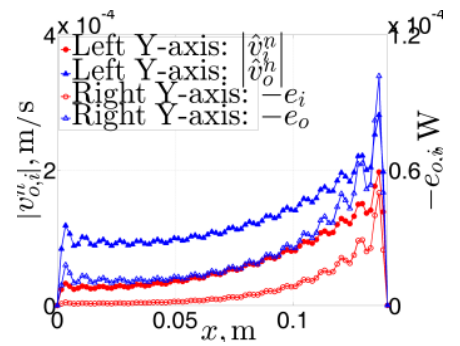

(b)
Figure 3. (Color online) Mode $1 \mathrm{~L} 1 \mathrm{~A} ; \mathrm{Lc} 1=0.01 \mathrm{~m}, \mathrm{~L}=0.14 \mathrm{~m}$. (a) Pressure mode shape in the combustor as well as in the backing cavities; (b) amplitudes of acoustic velocities and acoustic energy flux absorption per unit area at the perforated plates.

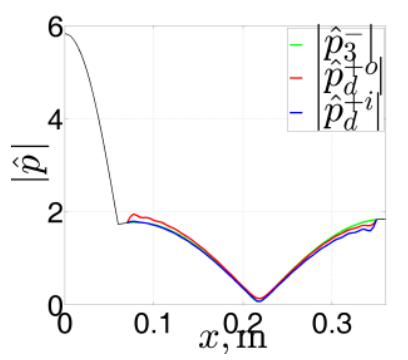

(a)

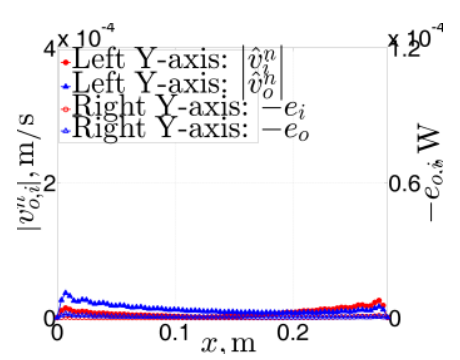

(b)
Figure 4. (Color online) Mode 1L1A; $L_{c 1}=0.01 \mathrm{~m}, \quad L=0.28$ m. (a) Pressure mode shape in the combustor as well as in the backing cavities; (b) amplitudes of acoustic velocities and acoustic energy flux absorption per unit area at the perforated plates.

Aiming at improving the control effect of the perforated liner when it is needed to occupy the whole simplified combustion chamber, we developed a technique by splitting the single backing cavity into two unconnected cavities with a rigid wall placed in the centre of the original backing cavities. As shown in Figure 5, the control effect is dramatically improved. The mechanism behind this is that the rigid wall inserted provides the backing cavities pressure anti-nodes that makes differences between the pressure oscillations in the main chamber and backing cavities larger, which is key to the acoustic damping the perforated plates. This can be seen in Figure 6.

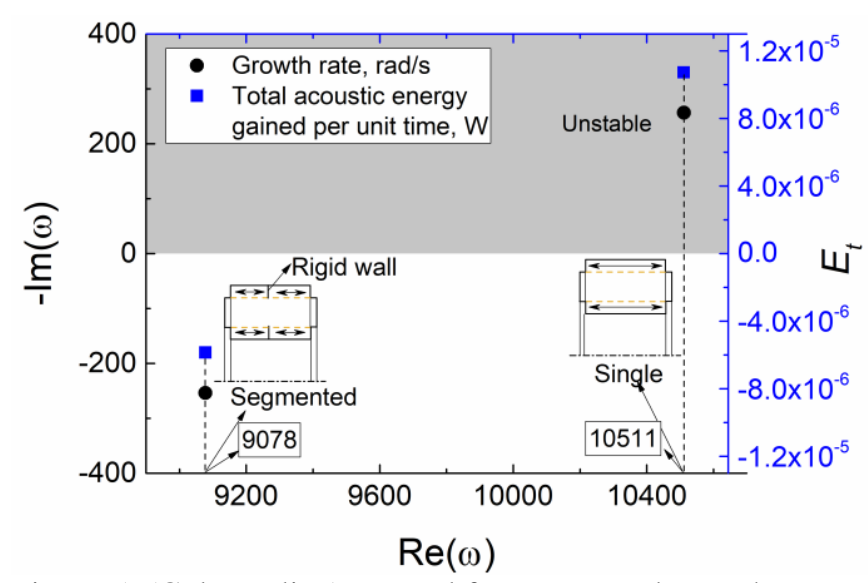

Figure 5. (Color online) Natural frequency and growth rate as well as the total acoustic energy flux gained for the two cases: the "Single" one and "Split" one.

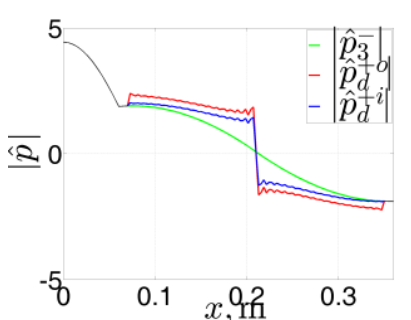

(a)

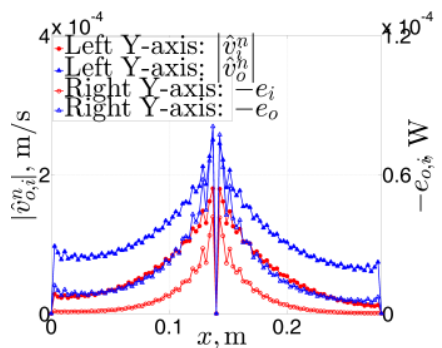

(b)
Figure 6. (Color online) Mode 1L1A; $L_{c 1}=0.01 \mathrm{~m}, \quad L=0.14$ m. (a) Pressure mode shape in the combustor as well as in the backing cavities; (b) amplitudes of acoustic velocities and acoustic energy flux absorption per unit area at the perforated plates.

\section{CONCLUSION}

In the present paper, we presented the theoretical calculations using TEM regarding the control effects of perforated liners on combustion instabilities. We found that the inclusion of the perforated plates with bias flow is helpful to the suppression of combustion instabilities on the condition that enough difference of pressure oscillations across the perforated plates is guaranteed. Specifically, when the perforated plates with backing cavities occupies the whole length of the combustion chamber, which is necessary for cooling purpose, the control effect will become insignificant. For this case, it can be dramatically improved by splitting the single backing cavity into two unconnected cavities with a rigid wall placed in the centre of the original backing cavities.

\section{ACKNOWLEDGMENTS}

This study was supported by National Natural Science Foundation of China (grant Nos. 11661141020 and 51676008). 


\section{REFERENCES}

[1] Akamatsu, S., \& Dowling, A. P. (2001, June). Three dimensional thermoacoustic oscillation in a premix combustor. In ASME Turbo Expo 2001: Power for Land, Sea, and Air (pp. V002T02A001-V002T02A001). American Society of Mechanical Engineers.

[2] Bourgouin, J. F., Durox, D., Moeck, J. P., Schuller, T., \& Candel, S. (2013, June). Self-sustained instabilities in an annular combustor coupled by azimuthal and longitudinal acoustic modes. In ASME Turbo Expo 2013: Turbine Technical Conference and Exposition (pp. V01BT04A007-V01BT04A007). American Society of Mechanical Engineers.

[3] Eldredge, J. D., \& Dowling, A. P. (2003). The absorption of axial acoustic waves by a perforated liner with bias flow. Journal of Fluid Mechanics, 485, 307-335.

[4] Howe, M. S. (1979). On the theory of unsteady high Reynolds number flow through a circular aperture. Proceedings of the Royal Society of London. A. Mathematical and Physical Sciences, 366(1725), 205223.

[5] Jing X, Sun X. Experimental investigations of perforated liners with bias flow[J]. The Journal of the Acoustical Society of America, 1999, 106(5): 2436-2441.

[6] Lieuwen, T. C., \& Yang, V. (Eds.). (2005). Combustion instabilities in gas turbine engines: operational experience, fundamental mechanisms, and modeling. American Institute of Aeronautics and Astronautics.

[7] Morfey, C. L. (1971). Acoustic energy in non-uniform flows. Journal of Sound and Vibration, 14(2), 159-170.

[8] Namba, M., \& Fukushige, K. (1980). Application of the equivalent surface source method to the acoustics of duct systems with non-uniform wall impedance. Journal of Sound and Vibration, 73(1), 125-146.

[9] Poinsot, T. (2017). Prediction and control of combustion instabilities in real engines. Proceedings of the Combustion Institute, 36(1), 1-28.

[10] Sun, X., Wang, X., Du, L., \& Jing, X. (2008). A new model for the prediction of turbofan noise with the effect of locally and non-locally reacting liners. Journal of Sound and Vibration, 316(1-5), 50-68.

[11] Wang, X., \& Sun, X. (2011). A new segmentation approach for sound propagation in non-uniform lined ducts with mean flow. Journal of Sound and Vibration, 330(10), 2369-2387.

[12] Worth, N. A., \& Dawson, J. R. (2013). Self-excited circumferential instabilities in a model annular gas turbine combustor: Global flame dynamics. Proceedings of the Combustion Institute, 34(2), 3127-3134.

[13] Zhang, G., Wang, X., Li, L., Jing, X., \& Sun, X. (2017). Control of thermoacoustic instability with a drum-like silencer. Journal of Sound and Vibration, 406, 253-276.

[14] Zhao, D., \& Li, X. Y. (2015). A review of acoustic dampers applied to combustion chambers in aerospace industry. Progress in Aerospace Sciences, 74, 114-130. 